\title{
Application of direct oral microscopy in evaluating mucosal margins around invasive oral squamous cell carcinoma
}

\author{
Piotr Chomik ${ }^{1,2}$, Adam Michcik ${ }^{1,2}$, Igor Michajłowski ${ }^{3,4}$, Anna Starzyńska ${ }^{1,2}$ \\ 1Department of Oral and Maxillofacial Surgery, Medical University of Gdansk, Gdansk, Poland \\ Head of the Department: Prof. Adam Włodarkiewicz MD, DMD, PhD \\ 2Department of Otolaryngology, Ward of Maxillofacial Surgery, University Clinical Center, Gdansk, Poland \\ Head of the Department: Prof. Adam Włodarkiewicz MD, DMD, PhD \\ ${ }^{3}$ Department of Dermatology, Venereology and Allergology, Medical University of Gdansk, Gdansk, Poland \\ Head of the Department: Prof. Roman Nowicki MD, PhD \\ ${ }^{4}$ Department of Dermatology, Venereology and Allergology, University Clinical Center, Gdansk, Poland \\ Head of the Department: Prof. Jadwiga Roszkiewicz MD, PhD
}

Postep Derm Alergol 2015; XXXII (5): 349-357

DOI: $10.5114 /$ pdia.2014.40792

\begin{abstract}
Introduction: Direct oral microscopy constitutes a novel, non-invasive diagnostic technique, which aids clinical examination of the oral cavity. The oral mucosa is examined at multiple magnifications and features such as subepithelial mucosal vessels, surface patterns, colour tone, transparency and the exact demarcation of mucosal lesions are estimated. The incidence of oral squamous cell carcinoma (OSCC) oscillates between 1.9\% and 3.5\%, which makes it the eighth most common carcinoma occurring around the world and in Poland. The 5-year survival rates oscillate between $20 \%$ and $30 \%$.

Aim: The aim of the study was to evaluate clinically unchanged mucosal margins around OSCC by direct oral microscopy. The authors approached the question whether the borders of mucosal margins around OSCC established via direct oral microscopy differ from those established based on clinical examination.

Material and methods: Fifteen patients diagnosed with OSCC were enrolled. Patients were first clinically examined to evaluate the extent of the tumour and to plan resection margins. Eventually, direct oral microscopy was performed to establish the width of the subclinically unchanged mucosal margins based on a standard picture of healthy oral mucosae, followed by comparison with those established by clinical evaluation.

Results: Histopathologic results of biopsies from areas indicated by direct oral microscopy revealed dysplasia in $86.7 \%$ of patients, whereas biopsies from areas indicated by clinical examination revealed dysplasia only in $40 \%$ of individuals, resulting in the need for widening of mucosal margins.

Conclusions: Direct oral microscopy enables detection of dysplasia within clinically unaltered mucosal margins around OSCC, which results in more precise establishing of resection boundaries, contributing to improvement of resection totality.
\end{abstract}

Key words: direct microscopy, oral cavity, squamous cell carcinoma.

\section{Introduction}

Direct oral microscopy constitutes a novel, as yet sporadically utilised, non-invasive diagnostic technique, which aids clinical examination of the oral cavity [1]. The basic principles of this method derive from colposcopy, a common diagnostic tool in gynaecology, and dermoscopy, which is utilised in clinical evaluations of skin lesions. The oral mucosa is examined microscopically at multi- ple magnifications so that features such as sub-epithelial mucosal vessels, surface patterns, colour tone and transparency as well as the exact demarcation boundaries of mucosal lesions can be estimated. The examination of sub-epithelial mucosal vessels is facilitated by the application of a green filter to the light source, which enhances the contrast between these vessels and the surrounding tissues [2].

Address for correspondence: Piotr Chomik DMD, PhD, Ward of Maxillofacial Surgery, University Clinical Center, 17 Smoluchowskiego St, 80-214 Gdansk, Poland, phone: +48 5834930 90, fax: +48 5834931 00, e-mail: piotr.chomik@gumed.edu.pl; wlodarkiewicz@gumed.edu.pl Received: 11.03.2014, accepted: 10.09.2014. 
According to the literature, the incidence of oral squamous cell carcinoma (OSCC) oscillates between 1.9\% and $3.5 \%$, which makes it the eighth most common carcinoma occurring around the world [3] and in Poland [4]. However, statistics concerning the treatment outcomes of the disease remain much more frightening. Despite its clinically accessible, non-diagnostically challenging primary location (oral cavity) as well as a wide variety of diagnostic tools and different schemes of surgical or systemic treatment or radiotherapy or combinations thereof, 5-year survival rates barely reach 20-30\% [4]. Various reasons are proposed to explain the problem. One of these is late diagnosis, after the tumour has already disabled normal functions of the oral cavity, causing patients' discomfort. Usually, by that point the disease has spread regionally, with metastases present in cervical lymph nodes. It is believed that such a condition primarily decreases the prognosis by 50\% [5]. However, subclinical and dysplastic lesions herald the development of invasive OSCC [6-8]. Correct diagnosis at that level enables definitive treatment of the disease, preserving normal function of the oral cavity, as well as avoiding aesthetic defects within the head and neck region.

Understanding the early stages of carcinogenesis in oral epithelium and typical clinical locations of cancers in the upper aerodigestive tract is not possible without commenting on the fields of cancerisation (FC) theory, described by Slaughter et al. [9] in 1953. First, it was applied to cancers in the head and neck region, including oral cancers, but further research revealed that it also relates to neoplasms in other anatomic regions, such as the larynx, lung, skin, breast and genital tract [10-12]. The basic principle of FC theory is the fact that oral cancer develops not from a single atypical dividing cell but its growth occurs in multiple locations simultaneously within precancerous lesions, and those locations (often recognized as independent foci of cancerous transformation) may eventually merge into one clinically visible primary tumour, or they may occur as separate multiple tumours at the same level of development when the treatment is started [11, 12].

What is more, according to FC theory principles and concordant clinical and histopathologic observations, the macroscopically healthy tissue between multiple tumours and tissue surrounding single primary tumours present histologically distinct grades of dysplasia, which also explains relatively frequent local recurrences or even so-called second primary tumours (SPT) that may occur even several years after histologically proven radical resection of a primary tumour [11, 12].

\section{Aim}

With regard to the above considerations, the aim of the study was to evaluate clinically unchanged mucosal margins dissected around invasive OSCC by direct oral microscopy according to colposcopic criteria: sub-epi- thelial mucosal vessels, surface patterns, colour tone, transparency and demarcation boundaries of mucosal margins. The authors approached the question whether the borders of mucosal margins around OSCC established via direct oral microscopy differ from those established based on clinical examination with the naked eye.

\section{Material and methods}

Adult patients of both genders, aged 46-81 (mean age: 63.5-year-old) referred to the Outpatient Office of Maxillofacial Surgery and the Ward of Maxillofacial Surgery in the Department of Otolaryngology, University Clinical Centre in Gdansk, Poland, due to invasive OSCC were included in the study. A total of 15 patients ( 9 males, 6 females) were examined and a special attention was given to estimation of healthy mucosal margins around invasive tumours. Every patient had histopathologically proven invasive OSCC.

The control group consisted of 15 patients of both genders (9 males, 6 females), aged 49-61 (mean age: 55-year-old), presenting clinically with unchanged oral mucosae, who were treated for trauma to the maxillofacial skeleton or cystic intra-bone tumours.

Information concerning general health, smoking habits, alcohol consumption and other addictions was collected for every patient.

Prior to commencing the study, every patient gave written consent to the clinical examination, direct oral microscopy and biopsies after having been informed about the procedures and aim of the study.

The study design was accepted by the Institutional Review Board of the Medical University in Gdansk (NKEBN/137/2011).

Patients diagnosed with invasive OSCC were first thoroughly examined under a halogen lamp with the naked eye to evaluate the extent of the tumour, the possible presence of satellite tumours and to plan resection margins. Eventually, direct oral microscopy was performed in a darkened room in order to establish the width of the subclinically unchanged mucosal margins based on a standard picture of healthy oral mucosae (control group), followed by a comparison with those established by clinical evaluation with the naked eye. Finally, the proper width of the mucosal margins around invasive OSCC was determined and surgery was performed. In direct oral microscopy the sub-epithelial mucosal vessels, surface patterns, colour tone and transparency as well as the exact demarcation boundaries of mucosal lesions based on the morphology of sub-epithelial capillaries were estimated.

All biopsy specimens obtained from mucosal margins after tumour resection were estimated in terms of dysplasia of oral squamous epithelium.

Post-resection specimens were evaluated in terms of the totality of the ablation and histopathologic disorders 
within mucosal margins, which were established based on both clinical examination with the naked eye and direct oral microscopy.

Patients from the control group were first thoroughly examined under a halogen lamp with the naked eye in order to confirm clinically unchanged oral mucosae. Direct oral microscopy was subsequently performed in order to establish the standard picture of healthy oral mucosae according to the colposcopic criteria mentioned above.

\section{Statistical analysis}

Differences in the intensity of particular features observed in the pictures were estimated according to an established numeric scale, where "O" meant the absence of the feature, "1" meant slight/weak presence of the feature, "2" indicated moderate intensity of the feature and " 3 " indicated a severe intensity of the described feature. Differences between particular groups of features or alterations visible in direct oral microscopy compared to unchanged oral mucosae were estimated using the Mann-Whitney $U$ test. The correlation between the most severely expressed feature via direct oral microscopy and the intensity of the remaining features was estimated using Spearman's range correlation ratio test ( $r$ parameter). Differences in the incidence of dysplasia between specimens collected based on direct oral microscopy and clinical examination were estimated using the test for the two indicators of structure. A $p$ value less than 0.05 was considered statistically significant. Statistical analysis was performed using Statistica $10 \mathrm{PL}$ software (StatSoft, Poland).

Direct oral microscopy was performed using a Leisegang colposcope, model BG/LED Y/C type 3ML (Leisegang, Germany). Photographs were taken with a Canon EOS 500D digital camera equipped with a Canon Macro

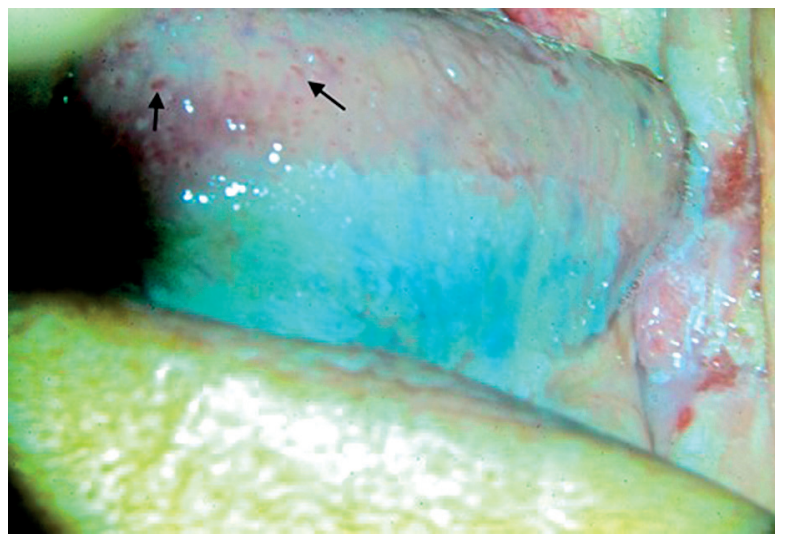

Figure 1. The arrows indicate punctation capillaries within clinically unchanged oral marginal mucosae around lingual cancer, seen with a green filter (magnification 15×)
100 mm lens (Canon, Japan). An Apple iMac computer with Mac OS X software (Apple, CA, USA) was utilised for the analysis, processing and archiving of pictures.

\section{Results}

In this study, direct oral microscopy was performed to examine clinically unchanged mucosal margins of invasive OSCC prior to resection in order to estimate their proper width preoperatively. Fifteen patients were examined utilizing colposcopic criteria to evaluate the oral mucosae surrounding the tumours. In all cases, abnormal patterns of sub-epithelial blood vessels were noted. Nine (60\%) patients revealed punctuated capillaries (Figure 1), whereas 6 (40\%) patients presented pathologic capillaries of the first degree according to Madej, which were branched, twisted and formed as a result of adaption of physiologic capillaries due to angiogenic factors produced by the tumour (Figure 2). According to colposcopic criteria, such a picture of sub-epithelial vessels heralds the presence of moderate to severe dysplasia or even already occurring malignant transformation.

All (100\%) patients presented with smooth, planar surface of mucosal margins around invasive OSCC, without features of hyperkeratinization and desquamation. Moreover, the surface of the mucosal margins was moist and shiny, similar to healthy oral mucosae (Figure 3).

The colour of mucosal margins was rosy to red. Among 15 cases, 8 (53.3\%) patients presented with rosy colour of marginal mucosae around invasive OSCC (Figure 4). The remaining 7 (46.7\%) patients revealed redness of mucosal margins around invasive tumours, most probably due to co-existing inflammation (Figure 5).

Marginal mucosa revealed partial to total transparency by direct oral microscopy. In 10 (66.7\%) patients, evaluation of sub-epithelial capillaries was possible without application of a green filter (Figure 6), whereas the

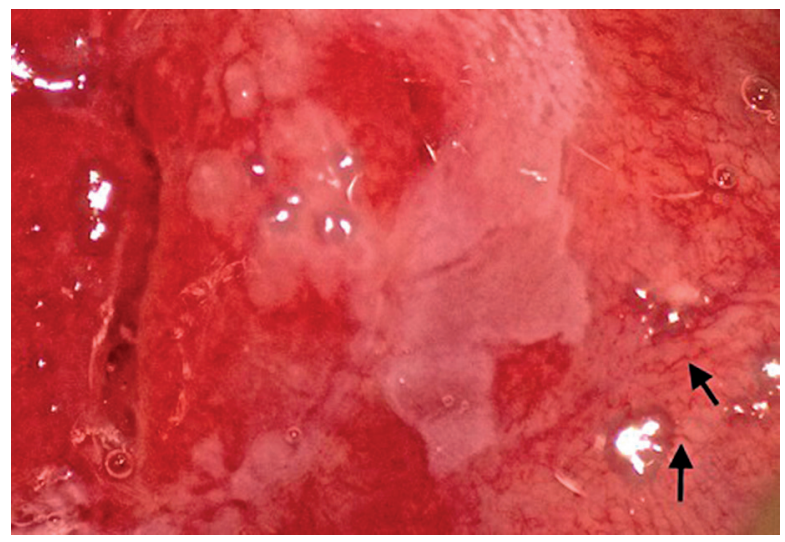

Figure 2. Branched pathologic vessels of the first degree according to Madej (arrows) visible within the margins of invasive buccal cancer (magnification 30x) 


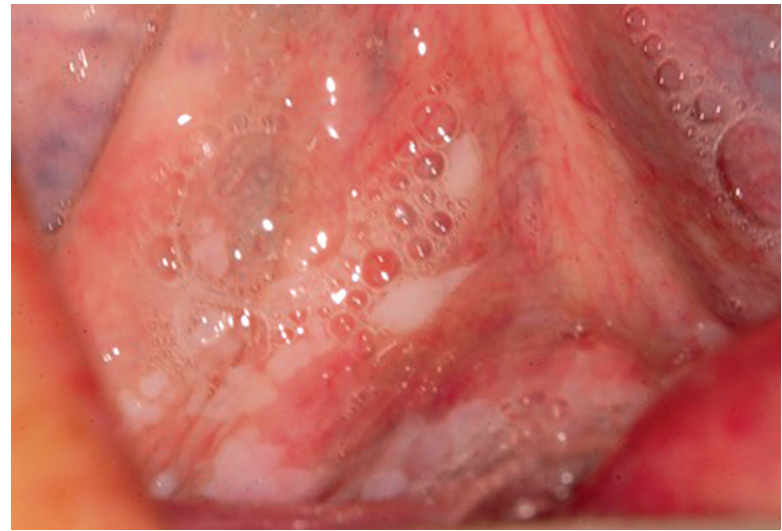

Figure 3. Smooth surface of marginal mucosae around invasive oral floor cancer. The moisture and gloss of the surface are similar to healthy oral mucosae (magnification 30x)

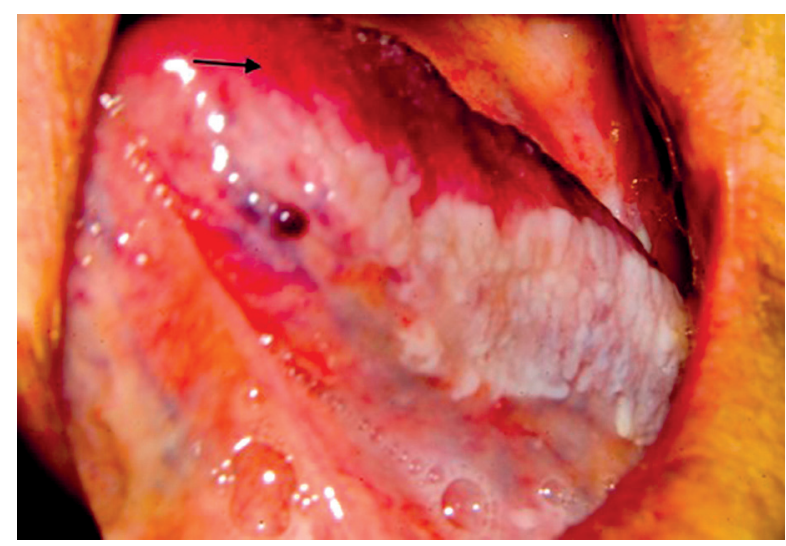

Figure 5. Lateral tongue cancer developed on the basis of long-term leucoplakia. The arrow indicates the redness of the marginal mucosa (magnification $7.5 \times$ )

remaining 5 (33.3\%) patients had sub-epithelial vessels that could be assessed using a green filter (Figure 7).

The boundaries of mucosal margins around invasive OSCC were described as areas, where subclinical mucosal disorders were not visible by direct oral microscopy according to colposcopic criteria. Clinically it was understood as a situation, when correct sub-epithelial capillary patterns appeared. Based on that condition, all (100\%) patients with invasive OSCC had mucosal margin boundaries described as irregular and unevenly proceeding into colposcopically unchanged oral mucosae (Figure 8).

Statistical analysis of the intensities of particular estimation parameters according to direct oral microscopy (sub-epithelial mucosal vessels, surface patterns, colour tone, transparency, exact demarcation boundaries) revealed significant differences from the established standard for oral mucosae except in terms of surface patterns $(p=0.99)$ and transparency $(p=0.21)$. The remaining parameters differed significantly from the established standard of oral mucosae via direct oral microscopy

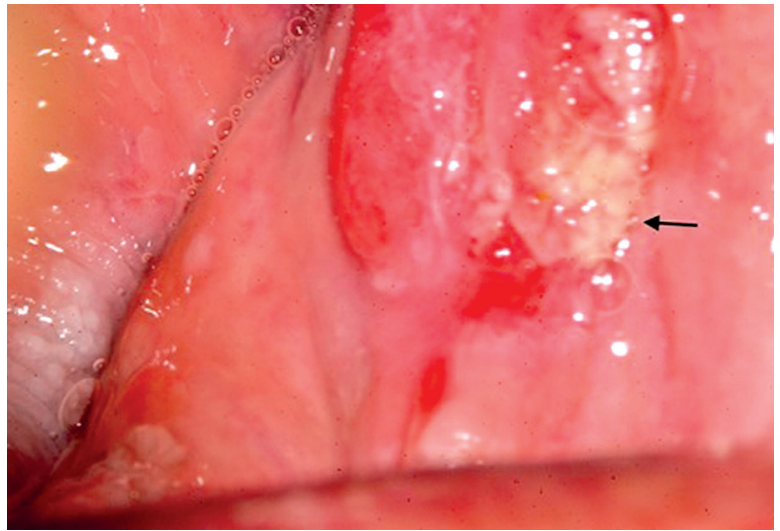

Figure 4. Mandibular gingival cancer (arrow). Rosy colour of marginal oral mucosae (magnification 30x)

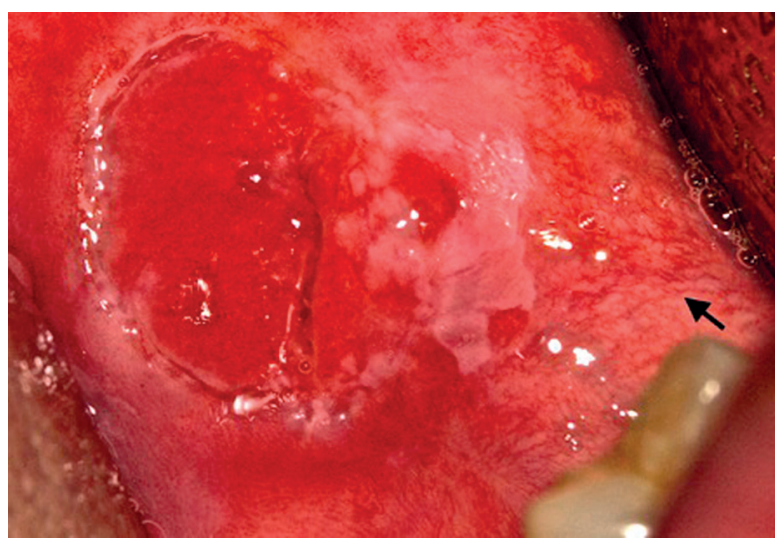

Figure 6. Total transparency of marginal mucosa around invasive buccal cancer. Sub-epithelial capillaries (arrow) visible without applying a green filter (magnification 15×)

( $p<0.05$ for each parameter). The combination of disorders within mucosal margins around invasive OSCC compared to the standard picture of oral mucosae evaluated by direct oral microscopy is presented in Table 1.

In 9 (60\%) out of 15 patients examined, histologic signs of moderate dysplasia were revealed in biopsies from mucosal margins around invasive tumours, whereas $4(26.7 \%)$ patients showed severe dysplasia, although the biopsied areas presented clinically as healthy oral mucosa (Figure 9). Biopsies were obtained from sites indicated by direct oral microscopy. In other words, 13 (86.7\%) out of 15 patients presented features of dysplasia within mucosal margins around invasive OSCC, which were established clinically with the naked eye (Table 2).

Compared to the results mentioned above, when biopsies were taken based on clinical evaluation with the naked eye, histologic features of moderate dysplasia were detected in 4 (26.7\%) individuals, whereas severe dysplasia was present in 2 (13.3\%) patients. In total, 40\% of patients presented histologic signs of dysplasia in 


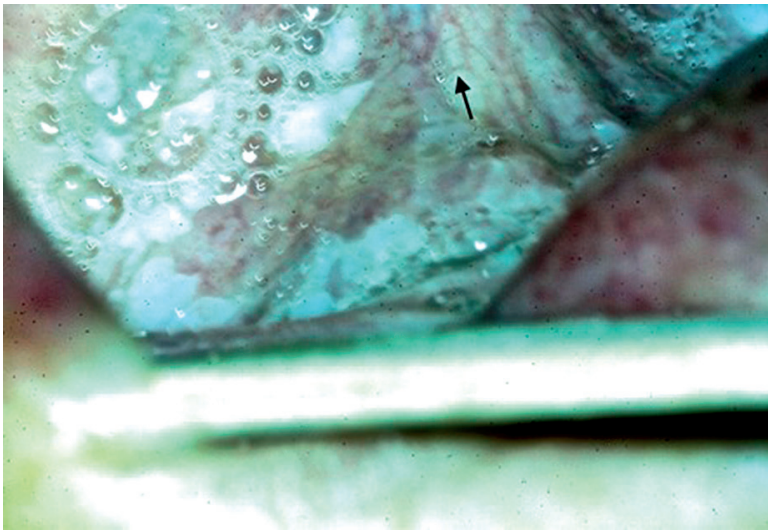

Figure 7. Partial transparency of marginal mucosa around invasive cancer of the ventral tongue. Sub-epithelial capillaries (arrow) within marginal mucosa as seen with a green filter (magnification 30x)

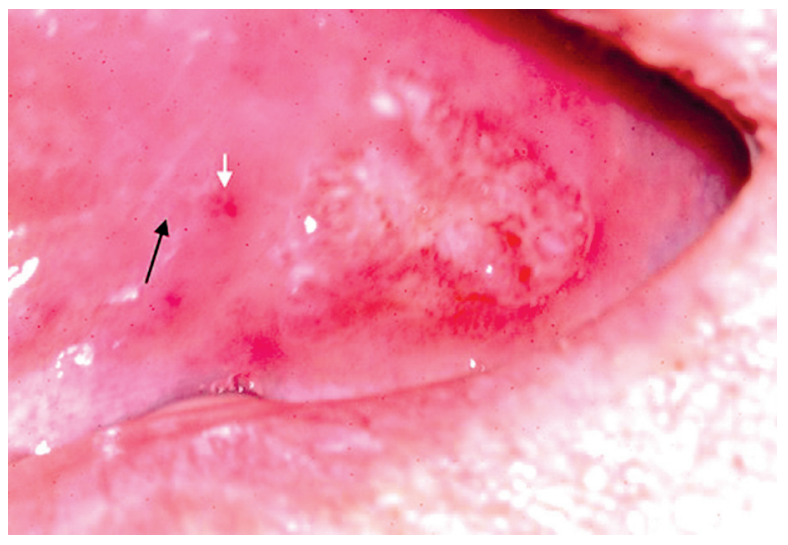

Figure 9. Lateral tongue cancer. A biopsy obtained from the site indicated by the black arrow revealed moderate dysplasia, while under clinical examination the site seemed healthy. Magnification $15 \times$ depicts focal redness of the mucosa in the biopsy site (grey arrow)

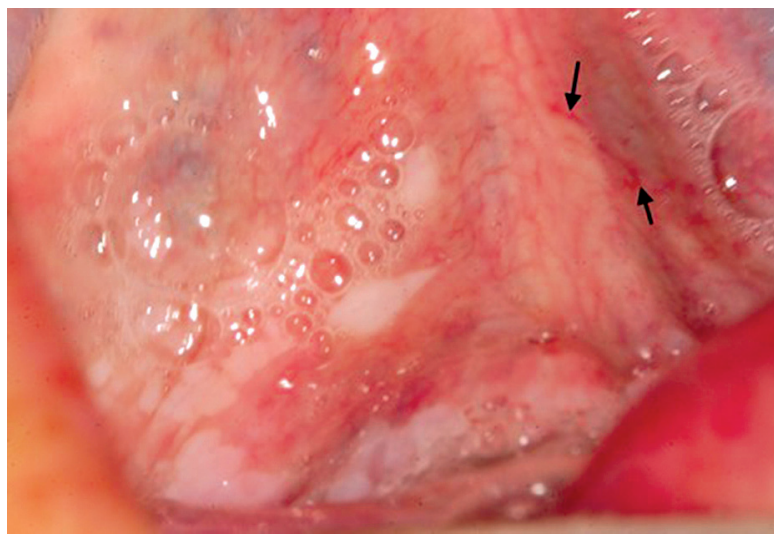

Figure 11. Extensively branched, pathologic vessels of the first degree according to Madej (arrows), seen within the mucosal margins around oral floor cancer (magnification 30x)

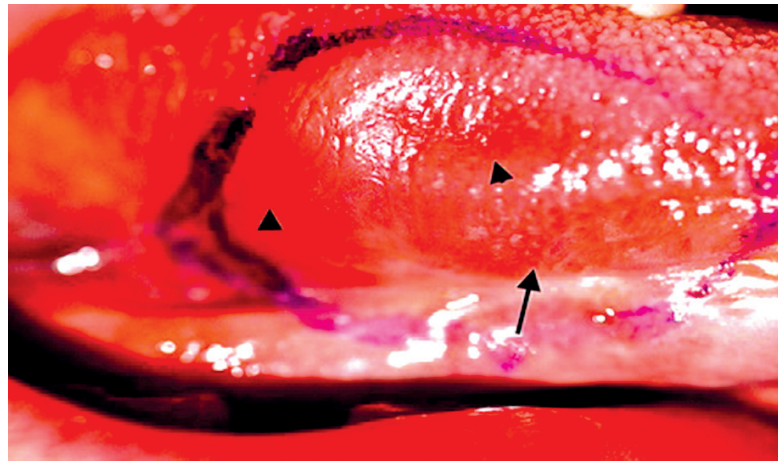

Figure 8. Local recurrence of lateral tongue cancer. Irregular boundaries of marginal mucosa around the tumour in the lower portion (arrow). Two foci of abnormal redness in the mucosa around the tumour (arrowheads) project to an unclear extent into the unaltered lingual mucosa. The violet line indicates the extent of resection (magnification 15x)

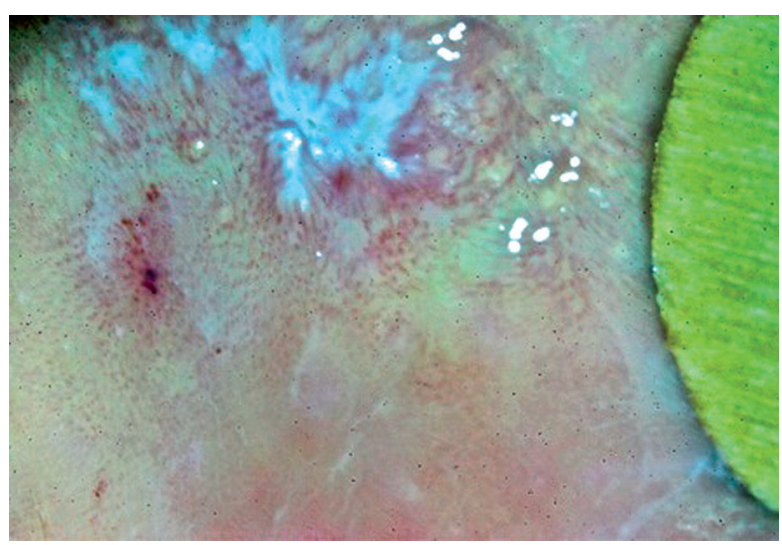

Figure 10. Numerous small punctation capillaries seen in the lower margins of a lesion diagnosed as homogenous leucoplakia, seen with a green filter (magnification 15×)

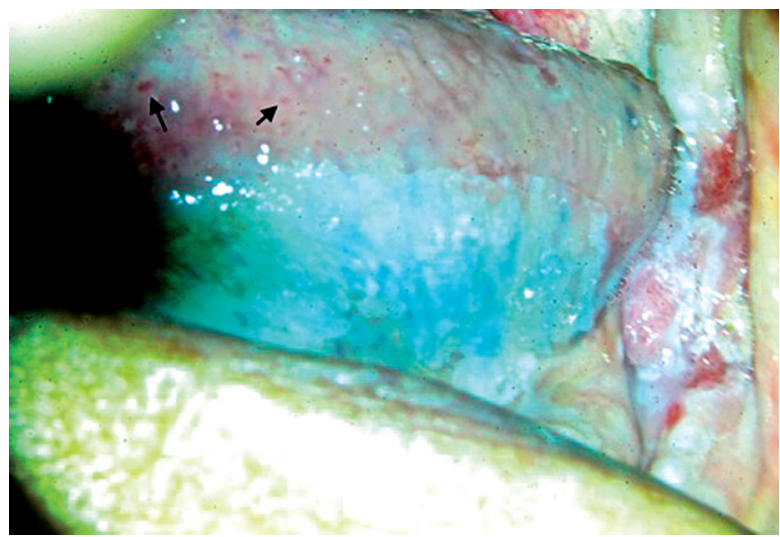

Figure 12. Arrows indicate sub-epithelial punctation capillaries within marginal mucosae around lateral tongue cancer (magnification 15x) 
Table 1. Combination of disorders within mucosal margins around invasive OSCC compared to the standard picture of oral mucosae via direct oral microscopy

\begin{tabular}{|c|c|c|}
\hline Feature & $\begin{array}{c}\text { Unaltered } \\
\text { oral mucosae }\end{array}$ & $\begin{array}{l}\text { Invasive OSCC } \\
\text { margins }\end{array}$ \\
\hline $\begin{array}{l}\text { Picture of sub-epithelial } \\
\text { vessels }\end{array}$ & $1 / 2$ & $\begin{array}{c}2 / 3 \\
(p=0.0000111277)\end{array}$ \\
\hline Punctation vessels & 0 & $\begin{array}{c}3 \\
(p=0.0000000647)\end{array}$ \\
\hline $\begin{array}{l}\text { First degree pathologic } \\
\text { vessels according to } \\
\text { Madej }\end{array}$ & 0 & $\begin{array}{c}2 \\
(p=0.0000000647)\end{array}$ \\
\hline $\begin{array}{l}\text { Moist, smooth, glossy } \\
\text { surface }\end{array}$ & $1 / 2$ & $\begin{array}{c}2 \\
(p=0.0000000647)\end{array}$ \\
\hline Flat surface & 1 & $\begin{array}{c}1 \\
(p=0.9903948387)\end{array}$ \\
\hline Rosy colour & $1 / 2$ & $\begin{array}{c}2 \\
(p=0.0195186834)\end{array}$ \\
\hline Red colour & 0 & $\begin{array}{c}1 \\
(p=0.0000000647)\end{array}$ \\
\hline Transparency & $2 / 3$ & $\begin{array}{c}2 / 3 \\
(p=0.2105660886)\end{array}$ \\
\hline Clear borders & 0 & $\begin{array}{c}1 \\
(p=0.0000000647)\end{array}$ \\
\hline \multicolumn{3}{|c|}{$\begin{array}{l}\text { Legends: } 0 \text { - feature lacking, } 1 \text { - mildly intense feature, } 2 \text { - moderately intense } \\
\text { feature, } 3-\text { strongly intense feature. Differences are statistically significant } \\
\text { when } p<0.05 \text {. }\end{array}$} \\
\hline \multicolumn{3}{|c|}{$\begin{array}{l}\text { Table 2. Combination of histopathologic results of biopsies } \\
\text { from mucosal margins around invasive OSCC obtained } \\
\text { based on direct oral microscopy }\end{array}$} \\
\hline Degree of dysplasia & Invasi & ive OSCC margins \\
\hline Moderate dysplasia & & $9 / 15(60 \%)$ \\
\hline Severe dysplasia & & 4/15 (26.7\%) \\
\hline Total & & 13/15 (86.7\%) \\
\hline
\end{tabular}

Table 3. Comparison of frequency of dysplasia in biopsies from mucosal margins around invasive OSCC obtained by direct oral microscopy or clinical evaluation with the naked eye

\begin{tabular}{lcc}
\hline Degree of dysplasia & Direct oral microscopy & Naked eye \\
\hline Moderate dysplasia & $9 / 15(60 \%)$ & $4 / 15(26.7 \%)$ \\
\hline Severe dysplasia & $4 / 15(26.7 \%)$ & $2 / 15(13.3 \%)$ \\
\hline Total & $13 / 15(86.7 \%)$ & $6 / 15(40 \%)$ \\
& $(p=0.0079)$ & $(p=0.0079)$ \\
\hline
\end{tabular}

Differences are statistically significant when $p<0.05$.

specimens obtained upon clinical examination with the naked eye. Comparison of the frequency of dysplasia in biopsies from mucosal margins around invasive OSCC obtained following direct oral microscopy or by clinical evaluation with the naked eye revealed a significant difference between these two methods, with direct oral microscopy being the more sensitive $(p=0.0079)$ (Table 3$)$.

Evaluation of the prognostic value of each colposcopic criterion in the oral mucosae examination with regard to dysplasia frequency revealed that estimation of subepithelial vessels proved to be the most reliable. Punctation vessels (Figure 10) and pathologic vessels of the first degree according to Madej (Figure 11) were most often observed around invasive tumours.

Direct oral microscopy of marginal mucosae around invasive OSCC revealed sub-epithelial vessels in all 15 (100\%) patients. Besides the punctation vessels (60\%) and pathologic vessels of the first degree according to Madej (40\%), mentioned above, another group of the latter (Figure 11) was observed at a considerable distance from the marginal mucosae in $12(80 \%)$ individuals. The remaining 3 (20\%) patients revealed a separate group of punctation vessels (Figure 12) in an area distant from the marginal mucosae. Spearman's range correlation ratio test showed a strong positive correlation between atypical sub-epithelial vessels observed around invasive tumours and the disorders of marginal mucosae seen by direct oral microscopy $(r=0.72 ; p<0.05)$.

\section{Discussion}

In vivo imaging techniques are increasingly popular in modern medicine. The constant progress in biomedical engineering technologies is generating more accurate results, which in turn improve the clinical evaluation of patients and histopathological specimen interpretation. To date, these methods have been successfully applied in specialities such as gynaecology and dermatology. Colposcopic examination together with dye testing is routinely performed to improve clinical gynaecological evaluations, and has been recognised as crucial in cases of incorrect or unsatisfactory cytology of the uterine cervix [13]. The long-term application of colposcopy in a wide range of gynaecologic diagnostic tools, as well as the availability of increasingly more sophisticated equipment, has contributed to the relatively high sensitivity and specificity of this diagnostic method [14].

Dermatoscopy (epiluminescence microscopy, ELM) has achieved a similar significance in dermatology. The particular effectiveness of this method was demonstrated in the diagnosis of melanocytic lesions, skin cancers and melanomas, as well as in clinical differentiation between benign skin lesions and those suspected of malignant transformation [15]. The use of dermatoscopy to diagnose oral mucosal melanocytic lesions has also been reported [16-19]. This method was shown to be particularly effective for estimating the borders of pathologic lesions [20].

Technological progress in recent years has resulted in the development of sophisticated tools dedicated to 
in vivo diagnostics of the oral cavity at high magnification. Reports describing in vivo imaging of oral mucosa in search of potentially malignant lesions are still rare, however. For this reason, interpretation of the results obtained in this work was based mainly on gynaecological and dermatological literature, because the state of medical knowledge concerning in vivo tissue imaging in these disciplines enables reliable conclusions.

In a group of 15 patients with histopathologically proven invasive OSCC, direct oral microscopy was applied to estimate clinically unchanged oral mucosae around tumours in order to establish the proper margins of resection. All patients presented sub-epithelial vessels with pathologic architecture in direct oral microscopy of the area of clinically unchanged oral mucosae. These were usually either punctation capillaries or pathologic vessels of the first degree according to Madej. The sizes of these vessels usually enabled their observation in white light; nevertheless, application of the green filter facilitated the accurate study of their morphology. In this group of patients, colposcopic pictures of invasive uterine cervix cancer served as a reference, with a special focus on subepithelial vessels around tumours. Punctation capillaries and dilated, twisted and extensively branched pathologic vessels of the first degree are, according to Madej, dominant in colposcopic pictures of uterine cervix cancer [21, 22]. In advanced cervical cancers, pathologic vessels of the second degree are also present [22, 23]. These are de novo emerging endothelial pipes or elongated capillaries, which are stimulated by angiogenic factors produced by the tumour. Such vessels are better evaluated in the green filter applied to the white light source.

The surface of the marginal mucosae around invasive OSCC was smooth in all patients, without any features of epithelial hyperkeratinization. In colposcopic pictures of cervical cancer, the marginal mucosa was also pathologically unchanged, with a smooth, non-folded surface, without features of epithelial hyperkeratinization and desquamation [24]. These findings are equivalent to the picture obtained by direct oral microscopy. The marginal mucosa was properly moist and shiny both by direct oral microscopy and in colposcopy, i.e., it was clinically healthy.

The colour of the marginal mucosae by direct oral microscopy was rosy to red. The red colour was probably a sign of co-existing inflammation, where underlying vessels were overloaded with blood and the underlay itself was filled with inflammatory exudation. Such a situation was observed in almost half of the patients (46.7\%). Comparing these observations to colposcopic pictures of cervical cancer marginal mucosae, the latter was pale rosy to rosy [25]. Hyperaemia of the underlying tissues and resulting redness of mucosae surrounding cervical tumours was stated in cases of co-existing colpitis [24].

In direct oral microscopy of marginal mucosae around invasive OSCC, total transparency of the mucosae was noted in most cases, so observation of sub-epithelial structures was possible in white light. Such a condition could also have been due to thinning of the squamous epithelium in that area. Only one third of the patients needed application of the green filter to evaluate subepithelial structures within oral marginal mucosae. Colposcopic evaluation of mucosal margins around invasive cervical cancers revealed a slightly decreased transparency in the cervical mucosae in its vaginal portion compared to oral marginal mucosae $[24,26]$. However, in some cases, observation of sub-epithelial structures in cervical mucosae is also possible without a green filter, thus reduced transparency in that area is not always evident. In cases where observation of sub-epithelial structures in cervical cancer margins was not possible in white light, application of a green filter remains helpful, most often revealing the pathologic vessels mentioned above $[23,24]$.

In order to establish the extent of mucosal margins around invasive OSCC, the correct picture of sub-epithelial vessels (network or hairpin capillaries) by direct oral microscopy was set as the exact placement of the boundaries. Based on that parameter, the shape of the border was irregular in all cases; however, in every case, margins established by direct oral microscopy were wider than those estimated based only on clinical examination. Colposcopic observations of cervical cancer enable very accurate estimation of tumour infiltration, especially when acetic acid is applied in the trial, which results in secondary whitening of the lesions in most cases [24, 27]. However, estimation of mucosal margins around cervical cancer infiltration becomes less evident when an accurate picture of subepithelial vessels (network or hairpin capillaries) is taken into consideration. Under such circumstances the irregular shapes of cervical mucosal margins and their unclear transition into a colposcopically correct picture of the vaginal portion of uterine cervix become evident [22]. In extensive exophytic cervical tumours, a colposcopic picture of healthy marginal mucosae may be impossible to obtain [22, 25]. Analysis of reports concerning the effectiveness of dermatoscopy in the diagnosis of genital tumours revealed that in cases of invasive vulvar tumours the marginal mucosa contain strongly branched, dilated and elongated pathologic vessels, similar to the margins of invasive OSCC seen with direct oral microscopy and cervical cancers seen in colposcopy. Based on these findings, the borders of mucosal margins around vulvar tumours present irregular shapes [28].

Statistical analysis concerning the intensity of particular estimation parameters in direct oral microscopy of mucosal margins around invasive OSCC compared to healthy oral mucosae revealed no differences only in the flatness of the surface and the transparency of the oral mucosae. The remaining parameters revealed statistical differences between marginal mucosae around invasive OSCC and healthy oral mucosae via direct oral micros- 
copy. This may constitute additional evidence for the advantage of direct oral microscopy over routine clinical examination for in vivo evaluation of tissues around invasive cancers.

Histopathologic evaluation of marginal mucosae specimens around invasive OSCC obtained from sites where direct oral microscopy revealed an atypical picture of sub-epithelial capillaries showed signs of epithelial dysplasia in $86.7 \%$ of patients. Moreover, the biopsied sites were clinically estimated as healthy mucosae and the boundaries of the resection were primarily planned within those areas. Such a strategy could result in retaining dysplastic oral epithelium after surgery, thus increasing the risk of local recurrence or development of second primary tumours. Numerous reports concerning the histopathologic presence of various grades of epithelial dysplasia in marginal clinically healthy mucosae around invasive OSCC are available in the literature. They are in accordance with Slaughter's theory of fields of cancerisation mentioned above [8-12, 29-33]. In light of this direct oral microscopy may become a useful tool in establishing borders between sub-clinically altered marginal oral mucosae - where it is extremely difficult to detect any disorders with the naked eye - and healthy tissue. In support of this idea, biopsies obtained from clinically unchanged marginal oral mucosae based on clinical examination with the naked eye revealed signs of moderate to severe epithelial dysplasia in only $40 \%$ of patients, i.e., less than half the rate noted in specimens based on direct oral microscopy; the difference was statistically significant. On the one hand, such results may constitute an additional reason to utilize direct oral microscopy to estimate the width of mucosal margins prior to tumour resection. On the other hand, these differences help to clarify that invasive OSCC should not be treated as a tumour with dimensions that can necessarily be measured by either clinical or contrast radiologic examination. The overall evaluation of the tumour's influence on oral tissues and the resulting treatment plan must consider Slaughter's theory of fields of cancerisation.

Assessment of the diagnostic value of particular estimation parameters adapted from colposcopy after examining oral mucosae by means of direct microscopy revealed that estimation of sub-epithelial vessels was the most useful and predictable. The therapeutic and prognostic meaning of that parameter in direct oral microscopy seemed to be the most significant in evaluating mucosal margins around invasive OSCC. Every patient presented either pathologic vessels of the first degree according to Madej or punctation vessels. Moreover, all patients had atypical sub-epithelial capillaries visible a considerable distance from the oral mucosal margins. These were pathologic vessels of the first degree according to Madej in $80 \%$ of patients and the remaining $20 \%$ of individuals presented punctation vessels. A strong positive statistical correlation was also observed between the presence of atypical sub-epithelial vessels in oral marginal mucosae and other disorders in the marginal mucosae seen by direct oral microscopy. This observation is concordant with the well-known and already discussed angiogenic action of invasive tumours. The picture of atypical sub-epithelial vessels a considerable distance from the mucosal margins of invasive OSCC may further confirm Slaughter's theory of fields of cancerisation and should result in increased oncologic vigilance, because second primary tumours may develop in those sites in the future. In the authors' opinion, this is another reason for accepting direct oral microscopy as a valuable tool for in vivo oral mucosae examination and subclinical detection of pathology. Comparing those observations to colposcopic pictures of invasive cervical cancers, the presence of pathologic sub-epithelial vessels around the tumours is evident. Those capillaries frequently spread through the entire vaginal portion of the uterine cervix, hindering depiction of colposcopically healthy mucosae. They usually present as punctation capillaries, pathologic vessels of the first and second degree according to Madej, where the latter constitute de novo in cases of advanced tumours [21, 22, 24]. The significant similarity between atypical vessels observed via direct oral microscopy around invasive OSCC and pathologic vessels observed by colposcopy in patients with invasive cervical cancers should also be noted.

\section{Conclusions}

The adaption of in vivo tissue imaging techniques that are commonly utilised in gynaecology and dermatology to diagnose oral mucosae constitutes a novel research field that aims to improve the diagnosis of oral pre-cancers and cancers and improve their treatment outcomes. The results obtained in this study strongly support that idea. The availability of precise diagnostic equipment, together with a growing social awareness concerning oral cavity health, raises the hope that these goals may be achieved in the near future.

\section{Conflict of interest}

The authors declare no conflict of interest.

\section{References}

1. Drogoszewska B, Chomik P, Polcyn A, Michcik A. Clinical diagnosis of oral erosive lichen planus by direct oral microscopy. Postep Derm Alergol 2014; 31: 222-8.

2. Gynther GW, Rozell B, Heimdahl A. Direct oral microscopy and its value in diagnosing mucosal lesions. A pilot study. Oral Surg Oral Med Oral Pathol Oral Radiol Endod 2000; 90: 164-70.

3. Xiumei W, Wenjing S, Jing B, et al. Growth inhibition induced by transforming growth factor beta1 in human oral squamous cell carcinoma. Mol Biol Rep 2009; 36: 861-9.

4. Strycharz M, Polz-Dacewicz M, Gołąbek W, et al. The epidemiologic analysis of 254 oral cancer cases from the Lublin 
region. Annales Universitatis Mariae Curie-Skłodowska, Lublin, Polonia, Vol. LXI, N 2, 114, Sectio D; 2006; 655-9.

5. Rigual NR, Wiseman SM. Neck dissection: current concepts and future directions. Surg Oncol Clin N Am 2004; 13: 151-66.

6. Poswillo D. Evaluation, surveillance and treatment of panoral leukoplakia. J Max Fac Surg 1975; 3: 205-11.

7. Girard KR, Strahs GR, Chung B. Panoral verrucous dysplasia. Report of a case treated with split - thickness skin graft. Oral Surg Oral Med Oral Pathol 1980; 50: 124-6.

8. Thomson PJ, Hamadah O. Cancerisation within the oral cavity: the use of "field mapping biopsies" in clinical management. Oral Oncol 2007; 43: 20-6.

9. Slaughter DP, Southwick HW, Smejkal W. "Field cancerization" in oral stratified squamous epithelium. Clinical implications of multicentric origin. Cancer 1953; 6: 963-8.

10. Mignogna MD, Fedele S, Lo Russo L, et al. Field cancerization in oral lichen planus. EJSO - Eur J Surg Oncol 2007; 33: 383-9.

11. Braakhuis BJM, Tabor MP, Kummer A, et al. A genetic explanation of Slaughter's concept of field cancerization: evidence and clinical implications. Cancer Res 2003; 63: 1727-30.

12. Braakhuis BJM, Tabor MP, Leemans CR, et al. Second primary tumors and field cancerization in oral and oropharyngeal cancer: molecular techniques provide new insights and definitions. Head Neck 2002; 24: 198-206.

13. Grubisić G, Klarić P, Jokanović L, et al. Diagnostic approach for precancerous and early invasive cancerous lesions of the uterine cervix. Collegium Anthropologicum 2009; 33: 1431-6.

14. Tan JHJ, Wrede CDH. New technologies and advances in colposcopic assessment. Best Pract Res Cl Ob 2011; 25: 667-77.

15. Argenziano G, Ferrara G, Francione S, et al. Dermoscopy the ultimate tool for melanoma diagnosis. Semin Cutan Med Surg 2009; 28: 142-8.

16. De Giorgi V, Massi D, Carli P. Dermoscopy in the management of pigmented lesions of the oral mucosa. Oral Oncol 2003; 39: 534-5.

17. Lin J, Koga H, Takata M, Saida T. Dermoscopy of pigmented lesions on mucocutaneous junction and mucous membrane. Br J Dermatol 2009; 161: 1255-61.

18. Mannone F, De Giorgi V, Cattaneo A, et al. Dermoscopic features of mucosal melanosis. Dermatol Surg 2004; 30: 1118-23.

19. Gencoglan G, Gerceker-Turk B, Kilinc-Karaarslan I, et al. Dermoscopic findings in Laugier-Hunziker syndrome. Arch Dermatol 2007; 143: 631-3.

20. Braun RP, Rabinovitz HS, Krischer J, et al. Dermoscopy of pigmented seborrheic keratosis: a morphological study. Arch Dermatol 2002; 138: 1556-60.

21. Klimek R (ed.). Ginaecology. PZWL, Warsaw 1982.

22. Kolstad P. Vascular changes in cervical intraepithelial neoplasia and invasive cervical carcinoma. Clin Obstet Gynecol 1983; 26: 938-48.

23. Florczak K. Cervical cancer. Colposcopy. Digital Medicine in the Future 2009.

24. Florczak, K, Emerich J, Żmudzińska E. Gdańsk colposcopic presentations. Multimedia atlas. Digital Medicine in the Future 2005.

25. Jeronimo J, Long R, Neve L, et al. Preparing digitized cervigrams for colposcopy research and education: determination of optimal resolution and compression parameters. JLGTD 2006; 10: 39-44.

26. Basta A, Szczudrawa A, Pityński K, Kolawa W. The value of colposcopy and computerised colposcopy in diagnosis and therapeutic management of $\mathrm{CIN}$ and early invasive cervical cancer in pregnant women. Ginekol Pol 2002; 73: 307-13.

27. Szczudrawa A, Komnata-Gajda G, Bereza K, Skucińska D. Colposcopic screening in the prophylaxis of cervical cancer. Prz Lek 1999; 56: 14-6.

28. Lazaridou E, Fotiadou C, Giannopoulou C, Ioannides D. Persistent erythematous lesion of the vulva: a diagnostic and treatment challenge. J Drugs Dermatol 2012; 11: 110-2.

29. Cooper MP, Braakhuis BJ, de Vries N, et al. A panel of biomarkers of carcinogenesis of the upper aerodigestive tract as potential intermediate endpoints in chemoprevention trials. Cancer 1993; 71: 825-30.

30. Bongers V, Snow GB, de Vries N, Braakhuis BJ. Potential early markers of carcinogenesis in the mucosa of the head and neck using exfoliative cytology. J Pathol 1996; 178: 284-9.

31. Shin DM, Ro JY, Hong WK, Hittelman WN. Dysregulation of epidermal growth factor receptor expression in premalignant lesions during head and neck tumorigenesis. Cancer Res 1994; 54: 3153-9.

32. Cruz IB, Meijer CJ, Snijders PJ, et al. p53 immunoexpression in non-malignant oral mucosa adjacent to oral squamous cell carcinoma: potential consequences for clinical management. J Pathol 2000; 191: 132-7.

33. Angadi PV, Savitha JK, Rao SS, Sivaranjini Y. Oral field cancerization: current evidence and future perspectives. Oral Maxillofac Surg 2012; 16: 171-80. 